\title{
ON THE EQUIVALENCE OF VISCOSITY SOLUTIONS AND WEAK SOLUTIONS FOR A QUASI-LINEAR EQUATION*
}

\author{
PETRI JUUTINEN ${ }^{\dagger}$, PETER LINDQVIST $^{\ddagger}$, AND JUAN J. MANFREDI ${ }^{\S}$
}

Abstract. We discuss and compare various notions of weak solution for the $p$-Laplace equation

$$
-\operatorname{div}\left(|\nabla u|^{p-2} \nabla u\right)=0
$$

and its parabolic counterpart

$$
u_{t}-\operatorname{div}\left(|\nabla u|^{p-2} \nabla u\right)=0 .
$$

In addition to the usual Sobolev weak solutions based on integration by parts, we consider the $p$-superharmonic (or $p$-superparabolic) functions from nonlinear potential theory and the viscosity solutions based on generalized pointwise derivatives (jets). Our main result states that in both the elliptic and the parabolic case, the viscosity supersolutions coincide with the potential-theoretic supersolutions.

Key words. $p$-Laplacian, viscosity solutions, $p$-superharmonic functions

AMS subject classifications. Primary, 35J70, 35K65; Secondary, 31B99, 35D05

PII. S0036141000372179

1. Introduction. The objective of this paper is to prove that the viscosity solutions of the $p$-Laplace equation

$$
-\operatorname{div}\left(|\nabla u|^{p-2} \nabla u\right)=0
$$

and its parabolic analogue coincide with the usual weak solutions, defined with the aid of test-functions under the integral sign. Our main result is that the viscosity supersolutions are the same as the so-called $p$-superharmonic functions, which are defined through a comparison principle in nonlinear potential theory. In the linear case $p=2$ the viscosity supersolutions of the Laplace equation $-\Delta u=0$ are merely the superharmonic functions in classical potential theory. This result and its parabolic counterpart are due to Lions and can be found, for example, in [FIT]. For related results for equations with measurable coefficients see [Je2], and for certain classes of nonlinear equations see [CKSS].

The $p$-Laplace equation is the Euler-Lagrange equation for the variational integral

$$
\int_{\Omega}|\nabla u(x)|^{p} d x
$$

*Received by the editors May 15, 2000; accepted for publication (in revised form) May 13, 2001; published electronically October 11, 2001. This paper was written while the authors were participating in the program "Potential Theory and Nonlinear Partial Differential Equations" at the Mittag-Leffler Institute in Stockholm.

http://www.siam.org/journals/sima/33-3/37217.html

${ }^{\dagger}$ Department of Mathematics, University of Jyväskylä, P.O.Box 35 (MaD), FIN-40351 Jyväskylä, Finland (peanju@math.jyu.fi). This author's work was supported by the Academy of Finland, project 41964.

${ }^{\ddagger}$ Department of Mathematics, Norwegian University of Science and Technology, N-7491 Trondheim, Norway (lqvist@math.ntnu.no).

${ }^{\S}$ Department of Mathematics, University of Pittsburgh, Pittsburgh, PA 15260-0001 (manfredi@ pitt.edu). This author's work was supported in part by NSF grant DMS-9970687 and by the MittagLeffler Institute. 
Here $1<p<\infty$ is a fixed exponent, the function $u$ is scalar valued, and $\Omega$ is a domain in the $n$-dimensional Euclidean space. Notice that in the case $p=2$ (the linear case) we have the Dirichlet integral and the Laplace equation

$$
-\Delta u=0 \text {. }
$$

The $p$-harmonic equation is the prototype of a class of quasi-linear equations in the form

$$
-\operatorname{div} \mathcal{A}_{p}(x, \nabla u(x))=0
$$

and it is fundamental in the nonlinear potential theory; cf. [HKM]. The $p$-harmonic operator $\operatorname{div}\left(|\nabla u|^{p-2} \nabla u\right)$ also appears in many contexts in physics: non-Newtonian fluids (dilatant fluids have $p>2$, and pseudoplastics have $1<p<2$ ), reactiondiffusion problems, nonlinear elasticity (torsional creep), glaceology $(p=4 / 3)$, and the thermal radiation of a hydrogen bomb (see [B]), just to mention a few applications.

We will study the mere notion of solutions, subsolutions, and supersolutions. There is something new to be said about this much-investigated basic topic in connection with the so-called viscosity solutions, a modern concept originating in the theory of Hamilton-Jacobi equations. The viscosity solutions have turned out to be indispensable in the case $p=\infty$ (not treated here; see [Je], [JLM]) and quite useful for $p$ finite when it comes to the pointwise interpretation of expedient identities involving second derivatives; cf. [LMS]. We begin with a brief discussion about different definitions of solution.

The solutions with continuous second derivatives (classical solutions) have the advantage of being easy to define: the equation

$$
|\nabla u|^{2} \Delta u+(p-2) \sum_{i, j=1}^{n} \frac{\partial u}{\partial x_{i}} \frac{\partial u}{\partial x_{j}} \frac{\partial^{2} u}{\partial x_{i} \partial x_{j}}=0
$$

has to hold at each point in the domain $\Omega$. However, when $p \neq 2$ this class of solutions is too restricted for the solvability of the Dirichlet problem. Roughly speaking, this is due to the fact that solutions may not, in general, be of class $C^{2}$ at points where $\nabla u=0$.

To assure the solvability of the Dirichlet boundary value problem, the p-harmonic functions are usually defined as (continuous) weak solutions in the Sobolev space $W^{1, p}$. This is the familiar situation with test-functions under the integral sign; see Definition 2.1 below. The uniqueness comes almost for free here. In other words, one has both existence and uniqueness for the Dirichlet boundary value problem.

The definition of viscosity solutions is based on pointwise evaluation of the $p$ harmonic operator

$$
\Delta_{p} u=\operatorname{div}\left(|\nabla u|^{p-2} \nabla u\right)
$$

though only for smooth test-functions $\varphi$; see section 2 below. The underlying phenomenon is that the classical (of class $C^{2}$ ) sub- and supersolutions are enough to determine the $p$-harmonic functions, although the latter often are less smooth. This is the content of Corollary 2.6, which states that the viscosity solutions ${ }^{1}$ are the $p$-harmonic functions. To the best of our knowledge, this is a new result.

\footnotetext{
${ }^{1}$ To indicate the dependence on the exponent $p$, they are called viscosity $p$-solutions below.
} 
We have also included a section on the so-called $p$-parabolic equation

$$
u_{t}-\operatorname{div}\left(|\nabla u|^{p-2} \nabla u\right)=0
$$

According to Corollary 4.5, its viscosity solutions ${ }^{2}$ are the $p$-parabolic functions (continuous weak solutions in a parabolic Sobolev space). The interpretation of this result requires some caution in the range $1<p<\frac{2 n}{n+2}$, because discontinuous "solutions" have to be ruled out. The proof of the equivalence of the parabolic definitions is simpler than in the elliptic situation. We end the paper with a brief discussion of an alternative definition, due to Ishii and Souganidis [IS], for parabolic viscosity solutions in the singular case $1<p<2$.

Finally, let us briefly indicate an application. It is to be expected that, at least under suitable conditions, the limit

$$
\lim _{t \rightarrow \infty} u(x, t)
$$

of a $p$-parabolic function is $p$-harmonic. Such a theorem has been proved in [Ju] with the viscosity technique, which is advantageous for convergence problems. Our Corollary 2.6 complements the result, making it possible to conclude that the viscosity $p$-solution, obtained as limit function, is $p$-harmonic.

2. Definitions. Let $\Omega$ denote a domain in $\mathbb{R}^{n}$. The Sobolev space $W^{1, p}(\Omega)$ consists of all functions $u \Omega \rightarrow[-\infty, \infty]$ that together with their distributional first derivatives

$$
\nabla u=\left(\frac{\partial u}{\partial x_{1}}, \frac{\partial u}{\partial x_{2}}, \ldots, \frac{\partial u}{\partial x_{n}}\right)
$$

are $p$-summable. The corresponding local space is denoted by $W_{\text {loc }}^{1, p}(\Omega)$.

DeFinition 2.1. We say that a continuous function $u \in W_{\text {loc }}^{1, p}(\Omega)$ is p-harmonic in $\Omega$ if

$$
\int_{\Omega}|\nabla u|^{p-2}\langle\nabla u, \nabla \varphi\rangle d x=0
$$

for every $\varphi \in C_{0}^{\infty}(\Omega)$. Here $1<p<\infty$.

By elliptic regularity theory the continuity is redundant in the definition. According to a theorem of Ural'tseva, in the case $p>2$, later extended by DiBenedetto and Lewis to all $p>1, u \in C_{\mathrm{loc}}^{1, \alpha}(\Omega)$; cf. [Ur], [DB], [Le].

Next we will define the $p$-superharmonic functions via a comparison principle as in [Li]. Notice immediately that the "fundamental solution"

$$
V(x)=|x|^{\frac{p-n}{p-1}}
$$

if $1<p<n$ and

$$
V(x)=\log \left(\frac{1}{|x|}\right)
$$

\footnotetext{
ater.

${ }^{2}$ To indicate the dependence on the exponent $p$, they are called parabolic viscosity $p$-solutions
} 
if $p=n$ is, indeed, $p$-superharmonic in $\mathbb{R}^{n}$ according to the definition below, although

$$
\int_{|x|<1}|\nabla V(x)|^{p} d x=\infty .
$$

Any reasonable definition has to include this example. This is taken into account in the following potential-theoretic definition.

DeFinition 2.2. The function $u \Omega \rightarrow(-\infty, \infty]$ is called $p$-superharmonic if

(i) $u$ is lower semicontinuous,

(ii) $u \neq \equiv$, and

(iii) $u$ satisfies the comparison principle on each subdomain $D \Subset \Omega$ : if $h \in C(\bar{D})$ is p-harmonic in $D$ and $u \geq h$ on $\partial D$, then $u \geq h$ in $D$.

We have used the notation $D \Subset \Omega$ to indicate that the closure of the domain $D$ is contained in $\Omega$. For $p=2$, this is the classical definition of superharmonic functions due to Riesz. Note that there are no requirements for the gradient $\nabla u$ in Definition 2.2. However, a locally bounded $p$-superharmonic function $u$ is actually in $W_{\text {loc }}^{1, p}(\Omega)$ and satisfies the inequality

$$
\int_{\Omega}|\nabla u|^{p-2}\langle\nabla u, \nabla \varphi\rangle d x \geq 0
$$

for every nonnegative test-function $\varphi \in C_{0}^{\infty}(\Omega)$. Moreover, the converse is also true for a lower semicontinuous function $u$ in $W_{\mathrm{loc}}^{1, p}(\Omega)$. We refer the reader to [Li] for this fact and more information about the definition and properties of $p$-superharmonic functions.

Needless to say, p-subharmonic functions are defined in an analogous way, so that $u$ is $p$-subharmonic if and only if $-u$ is $p$-superharmonic. A function $u$ is $p$ harmonic if and only if it is both $p$-superharmonic and $p$-subharmonic. For the sake of completeness, we mention the comparison principle in nonlinear potential theory; see $[\mathrm{HKM}],[\mathrm{Li}]$.

Comparison principle for $p$-subharmonic and $p$-superharmonic functions. Suppose that $\Omega$ is a bounded domain, that $u$ is $p$-subharmonic, and that $v$ is $p$-superharmonic in $\Omega$. If

$$
\limsup _{x \rightarrow z} u(x) \leq \liminf _{x \rightarrow z} v(x)
$$

for all $z \in \partial \Omega$ and if both sides of the inequality are not simultaneously $+\infty$ or $-\infty$, then $u \leq v$ in $\Omega$.

Let us now turn our attention to viscosity solutions. The notion of viscosity solutions requires that the expression

$$
\begin{aligned}
\Delta_{p} \varphi & =\operatorname{div}\left(|\nabla \varphi|^{p-2} \nabla \varphi\right) \\
& =|\nabla \varphi|^{p-4}\left[|\nabla \varphi|^{2} \Delta \varphi+(p-2) \sum_{i, j=1}^{n} \frac{\partial \varphi}{\partial x_{i}} \frac{\partial \varphi}{\partial x_{j}} \frac{\partial^{2} \varphi}{\partial x_{i} \partial x_{j}}\right]
\end{aligned}
$$

be evaluated pointwise for smooth functions $\varphi$. This is not a problem when $\nabla \varphi \neq 0$, but the critical points pose additional difficulties, especially in the range $1<p<2$. A standard way to deal with singular equations in the theory of viscosity solutions is to use suitable semicontinuous extensions of the operator; cf. [CGG], [CIL]. For 
the $p$-Laplacian this approach would allow some false "solutions." For example, $u \equiv$ constant would then solve

$$
-\Delta_{p} u(x)=f(x)
$$

in the viscosity sense for any continuous function $f$.

In the following definition, the pointwise evaluation of $\Delta_{p} \varphi$ is avoided when $\nabla \varphi=0$. This precaution has no bearing if $p \geq 2$. (Observe that the difficulty with critical points cannot be just defined away, and, in connection with the approximating equation $-\Delta_{p} v=\varepsilon$, isolated critical points have to be dealt with; see Lemma 3.2 below.) To further motivate the definition, we remark that a function $\varphi \in C^{2}(\Omega)$ that satisfies $-\Delta_{p} \varphi(x)=0$ when $\nabla \varphi(x) \neq 0$ (nothing being said about the possible critical points) is $p$-harmonic in $\Omega$. This new result is an immediate consequence of Corollary 2.6 below.

Definition 2.3. The function $u \Omega \rightarrow(-\infty, \infty]$ is called a viscosity $p$-supersolution if

(i) $u$ is lower semicontinuous,

(ii) $u \neq \equiv \infty$, and

(iv) whenever $x_{0} \in \Omega$ and $\varphi \in C^{2}(\Omega)$ are such that $u\left(x_{0}\right)=\varphi\left(x_{0}\right), u(x)>\varphi(x)$ for $x \neq x_{0}$, and $\nabla \varphi\left(x_{0}\right) \neq 0$, we have

$$
-\Delta_{p} \varphi\left(x_{0}\right) \geq 0 .
$$

Each point $x_{0}$ requires its own family of test-functions touching from below, which may very well be empty. It is not difficult to see that condition (iv) can be replaced by the following condition.

(v) The following comparison holds for each subdomain $D \Subset \Omega$ : let $\varphi \in C^{2}(\Omega)$ be such that $\nabla \varphi(x) \neq 0$ and $-\Delta_{p} \varphi(x)<0$ in $D$. If $u \geq \varphi$ on $\partial D$, then $u \geq \varphi$ in $D$.

In other words, the comparison is with respect to "smooth strict subsolutions" in Definition 2.3 and with respect to $p$-harmonic functions in Definition 2.2. Our main result, Theorem 2.5 below, guarantees that both definitions yield the same class of $u$ 's. We have come to a fundamental issue about the difference between conditions (iii) and (v). At first sight, condition (v) looks like (iii) in Definition 2.2, especially if one replaces the strict inequality $-\Delta_{p} \varphi(x)<0$ by $-\Delta_{p} \varphi(x) \leq 0$, which is possible a posteriori due to our results. The point is that the comparison in (iii) is with respect to $p$-harmonic functions that are not necessarily of class $C^{2}$, the regularity being merely $C^{1, \alpha}$, while (v) is restricted to $C^{2}$-functions. It is in doubt whether one can further restrict the comparison in (iii) to $p$-harmonic functions $h$ having continuous second derivatives.

An upper semicontinuous function $u$ is a viscosity $p$-subsolution if $-u$ is a viscosity $p$-supersolution. A viscosity solution of the equation $-\Delta_{p} u=0$ is both a viscosity $p$-supersolution and $p$-subsolution.

Remark 2.4. If $p \geq 2$, then $-\Delta_{p} \varphi(x)$ is well defined also at the critical points of $\varphi$, and there is no need to require in (iv) that the gradient of a test-function does not vanish at the point of touching. However, since it turns out that both versions of the definition give the same class of solutions, we have decided to use the one that works also in the singular case $1<p<2$.

THEOREM 2.5. Let $1<p<\infty$. In a given domain the $p$-superharmonic functions and the viscosity $p$-supersolutions are the same.

Corollary 2.6. Let $1<p<\infty$. A function is p-harmonic if and only if it is a viscosity p-solution. 
The proof of Theorem 2.5 has two parts. First, we must prove that $p$-superharmonic functions are viscosity $p$-supersolutions. This is a rather immediate consequence of the classical comparison principle for $p$-superharmonic and $p$-subharmonic functions. Second, we must show that viscosity $p$-supersolutions are $p$-superharmonic, that is, they satisfy the comparison principle with respect to $p$-harmonic functions. This is more delicate since the points at which the gradient vanishes present difficulties in the degenerate as well as in the singular case. Since this comparison principle may be of independent interest, we have stated it by itself. The proof is presented in section 3 below.

THEOREM 2.7 (the comparison principle). Let $\Omega \subset \mathbb{R}^{n}$ be a bounded domain, and assume that $u$ is a viscosity p-subsolution and $v$ is a viscosity $p$-supersolution in $\Omega$. If

$$
\limsup _{x \rightarrow z} u(x) \leq \liminf _{x \rightarrow z} v(x)
$$

for all $z \in \partial \Omega$ and if both sides of (2.2) are not simultaneously $\infty$ or $-\infty$, then $u \leq v$ in $\Omega$.

Proof of Theorem 2.5. Let us first assume that $u$ is $p$-superharmonic. To show that $u$ is a viscosity $p$-supersolution, we argue by contradiction and assume that there exists $x_{0} \in \Omega$ and $\varphi \in C^{2}(\Omega)$ such that $u\left(x_{0}\right)=\varphi\left(x_{0}\right), u(x)>\varphi(x)$ for all $x \neq x_{0}$, $\nabla \varphi\left(x_{0}\right) \neq 0$, and

$$
-\Delta_{p} \varphi\left(x_{0}\right)<0
$$

By continuity, there exists a radius $r>0$ such that

$$
\left\{\begin{aligned}
-\Delta_{p} \varphi(x) & <0 \\
\nabla \varphi(x) & \neq 0
\end{aligned}\right.
$$

for every $x \in B_{r}\left(x_{0}\right)$. Let

$$
m=\inf _{\left|x-x_{0}\right|=r}(u(x)-\varphi(x))>0,
$$

and define $\tilde{\varphi}=\varphi+m$. Then $\tilde{\varphi}$ is $p$-subharmonic in the open set $B_{r}\left(x_{0}\right)$. Since $\tilde{\varphi} \leq u$ on $\partial B_{r}\left(x_{0}\right)$, we obtain from the comparison principle for $p$-superharmonic and $p$-subharmonic functions that $\tilde{\varphi} \leq u$ in $B_{r}\left(x_{0}\right)$. However,

$$
\tilde{\varphi}\left(x_{0}\right)=\varphi\left(x_{0}\right)+m>u\left(x_{0}\right),
$$

which is a contradiction. Therefore, $u$ must be a viscosity $p$-supersolution.

For the converse implication, it is enough to check that (iii) holds for viscosity $p$-supersolutions. This, however, follows immediately from Theorem 2.7 after noticing that by the first half of the proof, every $p$-harmonic function is a viscosity $p$-solution.

3. Proof of the comparison principle. Let the functions $u$ and $v$ satisfy the assumptions in Theorem 2.7. We begin with some simplifications of the general situation.

First reduction (approximation by smooth domains). We may assume, without loss of generality, that the bounded domain $\Omega$ is smooth, the function $v \in C^{1, \alpha}(\bar{\Omega})$ is $p$-harmonic, and $u \leq v$ on $\partial \Omega$. 
To see this, let us first observe that by (2.2) we can find for any $\epsilon>0$ a smooth domain $D \Subset \Omega$ such that $u<v+\epsilon$ in $\Omega \backslash D$. By semicontinuity there is a function $\varphi \in C^{\infty}(\Omega)$ such that

$$
u<\varphi<v+\epsilon
$$

on $\partial D$. Next, let $h$ be the unique weak solution to the Dirichlet problem

$$
\begin{cases}-\Delta_{p} h=0 & \text { in } D, \\ h=\varphi & \text { on } \partial D .\end{cases}
$$

In other words, $h \in C(\bar{D}) \cap W^{1, p}(D)$ is $p$-harmonic in $D$. Since $D$ is regular, $h$ takes its prescribed continuous boundary values $\varphi$ in the classical sense.

We have

$$
u \leq h \leq v+\epsilon
$$

on $\partial D$. In fact, it is known that $h \in C^{1, \alpha}(\bar{D})$ (see [Lie]), but we prefer to give an argument which avoids this difficult boundary regularity result. The weaker local regularity $h \in C_{\mathrm{loc}}^{1, \alpha}(D)$ will suffice. To this end, we construct a regular domain $D_{1} \Subset D$ such that

$$
u-\epsilon \leq h \leq v+2 \epsilon
$$

on $\partial D_{1}$ and $u<v+2 \epsilon$ in $\Omega \backslash D_{1}$. Notice that now we have $h \in C^{1, \alpha}\left(\bar{D}_{1}\right)$, because $h \in C_{\text {loc }}^{1, \alpha}(D)$. If we assume the theorem for regular domains, we get

$$
u-\epsilon \leq h \leq v+2 \epsilon
$$

in the whole $D_{1}$. Therefore, we conclude that $u \leq v+3 \epsilon$ in $\Omega$. Since $\epsilon>0$ was arbitrary, this is the desired situation. Moreover, since the two cases $u-\epsilon \leq h$ and $h \leq v+2 \epsilon$ are symmetric, it suffices to prove that $u-\epsilon \leq h$ in $D_{1}$.

Second reduction (approximation by "regularized" equations). It is enough to prove the comparison principle in the case when $v$ is a weak solution of the equation

$$
-\Delta_{p} v=\varepsilon, \quad \varepsilon>0 .
$$

More precisely, suppose that $v$ is a weak solution of (3.1) with smooth boundary values $\left(v-w \in W_{0}^{1, p}(\Omega)\right.$ for some $\left.w \in C^{1, \alpha}(\bar{\Omega})\right)$ and $\Omega$ is a bounded smooth domain. If $u$ is a viscosity $p$-subsolution in $\Omega$ such that $u(x) \leq v(x)$ for all $x \in \partial \Omega$, then we have $u(x) \leq v(x)$ for $x \in \Omega$.

Indeed, let us assume that the comparison principle holds in the setting described above, and let $u$ and $v$ be as in the first reduction. If $v_{\varepsilon}$ is the unique weak solution of (3.1) with the boundary condition $v_{\varepsilon}=v$ on $\partial \Omega$, then by the assumed comparison $u \leq v_{\varepsilon}$ in $\Omega$ for every $\varepsilon>0$. On the other hand, by Lemma 3.1 below, $v_{\varepsilon} \rightarrow v$ locally uniformly. This, in turn, means that $u \leq v$ in $\Omega$, which is exactly what we want to prove.

Lemma 3.1. Let $v \in W^{1, p}(\Omega)$ be p-harmonic in a bounded domain $\Omega$, and let $v_{\varepsilon}$ be the unique weak solution of the Dirichlet problem

$$
\left\{\begin{aligned}
-\Delta_{p} v_{\varepsilon}=\varepsilon & \text { in } \Omega, \\
v_{\varepsilon}=v & \text { on } \partial \Omega .
\end{aligned}\right.
$$

Then $v_{\varepsilon} \rightarrow v$ locally uniformly in $\Omega$ as $\varepsilon \rightarrow 0$. 
Proof. Take $v-v_{\varepsilon} \in W_{0}^{1, p}(\Omega)$ as a test-function in (1.1) and (3.1), and subtract the resulting equations. This yields

$$
\begin{aligned}
\int_{\Omega}\left\langle|\nabla v|^{p-2} \nabla v-\left|\nabla v_{\varepsilon}\right|^{p-2} \nabla v_{\varepsilon}, \nabla v-\nabla v_{\varepsilon}\right\rangle d x & =\varepsilon \int_{\Omega}\left(v_{\varepsilon}-v\right) d x \\
& \leq \varepsilon|\Omega|^{\frac{p-1}{p}}\left(\int_{\Omega}\left|v-v_{\varepsilon}\right|^{p} d x\right)^{\frac{1}{p}} \\
& \leq K \varepsilon\left(\int_{\Omega}\left|\nabla v-\nabla v_{\varepsilon}\right|^{p} d x\right)^{\frac{1}{p}},
\end{aligned}
$$

where we have used the inequalities of Hölder and Sobolev, and $K=K(p, n, \Omega)$ is some constant depending only on $p, n$, and $\Omega$.

For $p \geq 2$, it follows easily from the elementary vector inequality [DB, Chapter I]

$$
|a-b|^{p} \leq 2^{p-1}\left\langle|a|^{p-2} a-|b|^{p-2} b, a-b\right\rangle
$$

that

$$
\int_{\Omega}\left|\nabla v-\nabla v_{\varepsilon}\right|^{p} d x \leq K \varepsilon^{\frac{p}{p-1}}
$$

where $K=K(p, n, \Omega)$. The singular case $1<p<2$ is slightly more delicate. Start with the vector inequality [DB, Chapter I]

$$
\frac{|a-b|^{2}}{(|a|+|b|)^{2-p}} \leq \gamma\left\langle|a|^{p-2} a-|b|^{p-2} b, a-b\right\rangle,
$$

where $\gamma$ depends only on $p$ and $n$ and $a, b \in \mathbb{R}^{n}$. By Hölder's inequality

$$
\int_{\Omega}\left|\nabla v-\nabla v_{\varepsilon}\right|^{p} d x \leq\left(\int_{\Omega} \frac{\left|\nabla v-\nabla v_{\varepsilon}\right|^{2}}{\left(|\nabla v|+\left|\nabla_{\varepsilon}\right|\right)^{2-p}} d x\right)^{\frac{p}{2}}\left(\int_{\Omega}\left(|\nabla v|+\left|\nabla v_{\varepsilon}\right|\right)^{p} d x\right)^{\frac{2-p}{2}}
$$

and this time

$$
\int_{\Omega} \frac{\left|\nabla v-\nabla v_{\varepsilon}\right|^{2}}{\left(|\nabla v|+\left|\nabla_{\varepsilon}\right|\right)^{2-p}} d x \leq \gamma K \varepsilon\left(\int_{\Omega}\left|\nabla v-\nabla v_{\varepsilon}\right|^{p} d x\right)^{\frac{1}{p}} .
$$

Therefore, we have the inequality

$$
\int_{\Omega}\left|\nabla v-\nabla v_{\varepsilon}\right|^{p} d x \leq K \varepsilon^{p}\left(\int_{\Omega}\left(|\nabla v|+\left|\nabla v_{\varepsilon}\right|\right)^{p} d x\right)^{2-p} .
$$

Since $\int_{\Omega}\left|\nabla v_{\varepsilon}\right|^{p} d x$, on the other hand, can be estimated in terms of $\int_{\Omega}|\nabla v|^{p} d x$ independently of $\varepsilon$ for $\varepsilon$ small, this implies

$$
\int_{\Omega}\left|\nabla v-\nabla v_{\varepsilon}\right|^{p} d x \leq C \varepsilon^{p}\left(1+\int_{\Omega}|\nabla v|^{p} d x\right)^{2-p} .
$$

By estimates (3.2), (3.3), it follows that we have a uniform bound for

$$
\int_{\Omega}\left|\nabla v_{\varepsilon}\right|^{p} d x
$$


independent of $\varepsilon$. It then follows from the interior regularity estimates for solutions of (3.1) (cf. [Le], [DB2]) that if we fix a compact set $K \subset \Omega$, we have a uniform bound for $\left\|v_{\varepsilon}\right\|_{C^{\alpha}(K)}$ for any $1<p<\infty$. By Ascoli-Arzelà's theorem, there exists a subsequence $\varepsilon_{i} \rightarrow 0$ for which $v_{\varepsilon_{i}} \rightarrow w$ uniformly in $K$. It follows again from (3.2), (3.3) that indeed $w=v$ and that the full sequence $v_{\varepsilon} \rightarrow v$ locally uniformly in $\Omega$ as $\varepsilon \rightarrow 0$.

The weak solutions of (3.1) can be seen as strict supersolutions of (1.1), and this property is of great importance in the proof below of the reduced version of Theorem 2.7. A similar type of approximation argument has been used by Jensen [Je] in connection with the $\infty$-Laplace equation.

We will need the following result on the "viscosity properties" of weak solutions of (3.1).

Lemma 3.2. Let $v_{\varepsilon} \in W^{1, p}(\Omega)$ be a continuous weak solution of the equation $-\Delta_{p} v_{\varepsilon}=\varepsilon$ in $\Omega$, and let $x_{0} \in \Omega$ and $\varphi \in C^{2}(\Omega)$ be such that $v_{\varepsilon}-\varphi$ has a strict local minimum at $x_{0}$. Then

$$
\limsup _{\substack{x \rightarrow x_{0} \\ x \neq x_{0}}}\left(-\Delta_{p} \varphi(x)\right) \geq \varepsilon
$$

provided that $\nabla \varphi\left(x_{0}\right) \neq 0$ or $x_{0}$ is an isolated critical point.

Remark. We have come to a decisive point. In the case $p \geq 2$ the proof yields that $-\Delta_{p} \varphi\left(x_{0}\right) \geq \varepsilon$ and that $\nabla \varphi(x) \neq 0$ in some neighborhood of $x_{0}$. It is the case $1<p<2$ that requires caution, because $-\Delta_{p} \varphi(x)$ is undetermined at the critical points (which may be encountered).

Proof. Suppose that the assertion is not true, that is, there is $r>0$ such that

$$
\nabla \varphi(x) \neq 0 \text { and }-\Delta_{p} \varphi(x)<\varepsilon
$$

when $0<\left|x-x_{0}\right|<r$. After a translation, we may assume that $x_{0}=0$. Take any nonnegative test-function $\phi \in C_{0}^{\infty}\left(B_{r}\right)$, and integrate over the annulus $\rho<|x|<r$. (The auxiliary $\rho>0$ can be skipped if $\nabla \varphi(0) \neq 0$.) According to Gauss's theorem,

$$
\begin{aligned}
-\oint_{|x|=\rho} \phi|\nabla \varphi|^{p-2}\left\langle\nabla \varphi, \frac{x}{\rho}\right\rangle d S & =\int_{\rho<|x|<r} \operatorname{div}\left(\phi|\nabla \varphi|^{p-2} \nabla \varphi\right) d x \\
& =\int_{\rho<|x|<r}|\nabla \varphi|^{p-2}\langle\nabla \varphi, \nabla \phi\rangle d x+\int_{\rho<|x|<r} \phi\left(\Delta_{p} \varphi\right) d x .
\end{aligned}
$$

The flux approaches 0 as $\rho \rightarrow 0_{+}$. Indeed,

$$
\left.\left|\oint_{|x|=\rho} \phi\right| \nabla \varphi\right|^{p-2}\left\langle\nabla \varphi, \frac{x}{\rho}\right\rangle d S \mid \leq\|\phi\|_{\infty}\|\nabla \varphi\|_{\infty}^{p-1} \omega_{n-1} \rho^{n-1}
$$

where $\omega_{n-1} \rho^{n-1}$ is the area of the sphere of radius $\rho$. By the antithesis we have

$$
\int_{\rho<|x|<r} \phi\left(\Delta_{p} \varphi\right) d x \geq-\varepsilon \int_{\rho<|x|<r} \phi d x \geq-\varepsilon \int_{B_{r}} \phi d x .
$$

Therefore, we obtain

$$
\int_{B_{r}}|\nabla \varphi|^{p-2}\langle\nabla \varphi, \nabla \phi\rangle d x=\lim _{\rho \rightarrow 0} \int_{\rho<|x|<r}|\nabla \varphi|^{p-2}\langle\nabla \varphi, \nabla \phi\rangle d x \leq \varepsilon \int_{B_{r}} \phi d x .
$$


Thus $\varphi$ is a weak subsolution of (3.1). We finish by using the comparison principle as in the proof of Theorem 2.5 presented in section 2 .

After the reductions made above, it suffices to prove the following version of the comparison principle [see (3.4) below]. In the proof we use the notation

$$
F(\eta, X)=-|\eta|^{p-2}\left[\operatorname{trace}(X)+(p-2)\left\langle X \frac{\eta}{|\eta|}, \frac{\eta}{|\eta|}\right\rangle\right]
$$

when $\eta \neq 0$ is a vector in $\mathbb{R}^{n}$ and $X \in S_{n}$, where $S_{n}$ denotes the class of real symmetric $n \times n$ matrices. For a smooth function $\varphi$ we clearly have

$$
F\left(\nabla \varphi(x), D^{2} \varphi(x)\right)=-\operatorname{div}\left(|\nabla \varphi(x)|^{p-2} \nabla \varphi(x)\right)
$$

when $\nabla \varphi(x) \neq 0$. Here $D^{2} \varphi=\left(\frac{\partial^{2} \varphi}{\partial x_{i} \partial x_{j}}\right)_{n \times n}$ is the Hessian matrix of $\varphi$.

Proposition 3.3. Suppose that $\Omega \subset \mathbb{R}^{n}$ is a smoothly bounded domain, $u$ is a viscosity $p$-subsolution, and $v \in C^{1, \alpha}(\bar{\Omega})$ is a weak solution of $-\Delta_{p} v=\varepsilon$ in $\Omega$ such that $u \leq v$ on $\partial \Omega$. Then $u \leq v$ in $\Omega$.

Proof. Without loss of generality, we may assume that $\varepsilon=1$. We argue by contradiction and assume that $u-v$ has an interior maximum, that is,

$$
\sup _{\Omega}(u-v)>\sup _{\partial \Omega}(u-v)
$$

Consider the functions

$$
w_{j}(x, y)=u(x)-v(y)-\Psi_{j}(x, y), \quad j=1,2, \ldots,
$$

where

$$
\Psi_{j}(x, y)=\frac{j}{q}|x-y|^{q}, \quad q>\max \left\{\frac{p}{p-1}, 2\right\},
$$

and let $\left(x_{j}, y_{j}\right)$ be a maximum of $w_{j}$ relative to $\bar{\Omega} \times \bar{\Omega}$. By (3.5) and Proposition 3.7 in [CIL], we see that for $j$ sufficiently large, $\left(x_{j}, y_{j}\right)$ is an interior point. Since

$$
u(x)-v(y)-\Psi_{j}(x, y) \leq u\left(x_{j}\right)-v\left(y_{j}\right)-\Psi_{j}\left(x_{j}, y_{j}\right)
$$

for all $x, y \in \Omega$, we obtain by choosing $x=x_{j}$ that

$$
v(y) \geq-\Psi_{j}\left(x_{j}, y\right)+v\left(y_{j}\right)+\Psi_{j}\left(x_{j}, y_{j}\right)
$$

for all $y \in \Omega$. Let us denote

$$
\phi_{j}(y)=-\Psi_{j}\left(x_{j}, y\right)+v\left(y_{j}\right)+\Psi_{j}\left(x_{j}, y_{j}\right)-\frac{1}{q+1}\left|y-y_{j}\right|^{q+1} .
$$

Then, clearly, $v-\phi_{j}$ has a strict local minimum at $y_{j}$, and thus

$$
\limsup _{\substack{y \rightarrow y_{j} \\ y \neq y_{j}}}\left(-\Delta_{p} \phi_{j}(y)\right) \geq 1
$$

by Lemma 3.2. This implies that $x_{j} \neq y_{j}$. Indeed, if $x_{j}=y_{j}$, then a direct computation shows that $-\Delta_{p} \phi_{j}(y) \rightarrow 0$ as $y \rightarrow y_{j}$, which is a contradiction. 
The rest of the proof is now a rather standard application of the maximum principle for semicontinuous functions (also known as the theorem on sums) in [CIL]. Since $\left(x_{j}, y_{j}\right)$ is a local maximum point of $w_{j}(x, y)$, we conclude that there exist symmetric $n \times n$ matrices $X_{j}, Y_{j}$ such that

$$
\begin{aligned}
\left(D_{x} \Psi_{j}\left(x_{j}, y_{j}\right), X_{j}\right) & \in \bar{J}^{2,+} u\left(x_{j}\right), \\
\left(-D_{y} \Psi_{j}\left(x_{j}, y_{j}\right), Y_{j}\right) & \in \bar{J}^{2,-} v\left(y_{j}\right),
\end{aligned}
$$

and

$$
\left(\begin{array}{cc}
X_{j} & 0 \\
0 & -Y_{j}
\end{array}\right) \leq D^{2} \Psi_{j}\left(x_{j}, y_{j}\right)+\frac{1}{j}\left[D^{2} \Psi_{j}\left(x_{j}, y_{j}\right)\right]^{2}
$$

Here $\bar{J}^{2,+} u\left(x_{j}\right)$ and $\bar{J}^{2,-} v\left(y_{j}\right)$ are the closures of the second order superjet of $u$ at $x_{j}$ and the second order subjet of $v$ at $y_{j}$, respectively. We refer the reader to $[\mathrm{C}]$ and [CIL] for the definition and properties of jets. that

Observe that since $D^{2} \Psi_{j}$ annihilates vectors of the form $\left(\begin{array}{c}\xi \\ \xi\end{array}\right)$, we obtain from (3.6)

$$
X_{j} \leq Y_{j}
$$

in the sense of matrices, that is, $\left\langle\left(Y_{j}-X_{j}\right) \xi, \xi\right\rangle \geq 0$ for all $\xi \in \mathbb{R}^{n}$.

Let us now finish the proof. It is well known (see [CIL]) that for equations that are continuous in each variable, viscosity solutions can be defined using jets instead of test-functions as in Definition 2.3. Since $x_{j} \neq y_{j}$, we have that

$$
\eta_{j} \equiv D_{x} \Psi_{j}\left(x_{j}, y_{j}\right)=-D_{y} \Psi_{j}\left(x_{j}, y_{j}\right) \neq 0
$$

This means that

$$
(\eta, X) \mapsto F(\eta, X)
$$

where $F$ is given by (3.4) and is continuous in a neighborhood of the points $\left(\eta_{j}, X_{j}\right)$ and $\left(\eta_{j}, Y_{j}\right)$, and we may use the equivalent definition involving jets. Since $u$ is a subsolution of (1.1), we obtain that

$$
-\left|\eta_{j}\right|^{p-2}\left[\operatorname{trace}\left(X_{j}\right)+(p-2)\left\langle X_{j} \frac{\eta_{j}}{\left|\eta_{j}\right|}, \frac{\eta_{j}}{\left|\eta_{j}\right|}\right\rangle\right] \leq 0 .
$$

On the other hand, since $\eta_{j} \neq 0$, by the definition of $\bar{J}^{2,-}$ Lemma 3.2 implies that

$$
-\left|\eta_{j}\right|^{p-2}\left[\operatorname{trace}\left(Y_{j}\right)+(p-2)\left\langle Y_{j} \frac{\eta_{j}}{\left|\eta_{j}\right|}, \frac{\eta_{j}}{\left|\eta_{j}\right|}\right\rangle\right] \geq 1
$$

Hence

$$
\begin{aligned}
0<1 \leq & -\left|\eta_{j}\right|^{p-2}\left[\operatorname{trace}\left(Y_{j}\right)+(p-2)\left\langle Y_{j} \frac{\eta_{j}}{\left|\eta_{j}\right|}, \frac{\eta_{j}}{\left|\eta_{j}\right|}\right\rangle\right] \\
& +\left|\eta_{j}\right|^{p-2}\left[\operatorname{trace}\left(X_{j}\right)+(p-2)\left\langle X_{j} \frac{\eta_{j}}{\left|\eta_{j}\right|}, \frac{\eta_{j}}{\left|\eta_{j}\right|}\right\rangle\right] \\
\leq & 0
\end{aligned}
$$


where the last inequality follows from the fact $X_{j} \leq Y_{j}$. This contradiction means that our initial assumption (3.5) cannot hold, and, therefore,

$$
\sup _{\Omega}(u-v)=\sup _{\partial \Omega}(u-v) \leq 0
$$

as claimed.

4. The parabolic case. The $p$-parabolic equation

$$
u_{t}-\operatorname{div}\left(|\nabla u|^{p-2} \nabla u\right)=0,
$$

where $u=u(x, t)$, has the $p$-harmonic equation as its stationary equation. Let us introduce some notation. Let

$$
Q=\left(a_{1}, b_{1}\right) \times\left(a_{2}, b_{2}\right) \times \cdots \times\left(a_{n}, b_{n}\right)
$$

denote a parallelepiped, and consider the "space-time box"

$$
Q_{t_{1}, t_{2}}=Q \times\left(t_{1}, t_{2}\right)
$$

in the $(x, t)$-space. Its parabolic boundary is

$$
\partial_{\text {par }} Q=\left(\bar{Q} \times\left\{t_{1}\right\}\right) \cup\left(\partial Q \times\left(t_{1}, t_{2}\right]\right) .
$$

It consists of the bottom and the lateral sides, but the interior points of the top are excluded.

In order to describe the appropriate function space, we introduce the abbreviation

$$
V^{p}\left(t_{1}, t_{2} ; Q\right)=C\left(t_{1}, t_{2} ; L^{2}(Q)\right) \cap L^{p}\left(t_{1}, t_{2} ; W^{1, p}(Q)\right) .
$$

Thus $u \in V^{p}\left(t_{1}, t_{2} ; Q\right)$ implies that the mapping

$$
t \mapsto \int_{Q}|u(x, t)|^{2} d x
$$

is continuous in $\left[t_{1}, t_{2}\right]$, the Sobolev derivative

$$
\nabla u(x, t)=\left(\frac{\partial u(x, t)}{\partial x_{1}}, \ldots, \frac{\partial u(x, t)}{\partial x_{n}}\right)
$$

exists for almost every $t$ in $\left[t_{1}, t_{2}\right]$, and the integral

$$
\int_{t_{1}}^{t_{2}} \int_{Q}\left(u^{2}+|\nabla u|^{p}\right) d t d x
$$

is finite.

Definition 4.1. Let $\mathcal{O}$ be a domain in $\mathbb{R}^{n} \times \mathbb{R}$, and suppose that the function $u \mathcal{O} \rightarrow \mathbb{R}$ is continuous and belongs to $V^{p}\left(t_{1}, t_{2} ; Q\right)$ whenever the closure of $Q_{t_{1}, t_{2}}$ is comprised in $\mathcal{O}$. We say that $u$ is p-parabolic in $\mathcal{O}$ if

$$
\iint_{\mathcal{O}}\left(-u \varphi_{t}+|\nabla u|^{p-2}\langle\nabla u, \nabla \varphi\rangle\right) d t d x=0
$$

for all test-functions $\varphi \in C_{0}^{\infty}(\mathcal{O})$. 
By parabolic regularity theory, the continuity is a redundant requirement in the definition if $p>\frac{2 n}{n+2}$. The case $1<p \leq \frac{2 n}{n+2}$ is not fully understood. It is known that there exist locally unbounded $u$ 's satisfying (4.1) in a weaker sense than described above. We refer to [DB, Chapter XII]. Such weak solutions cannot be viscosity solutions, since the latter are both upper and lower semicontinuous by definition and hence locally bounded. Thus our characterization of the $p$-parabolic viscosity solutions discards such discontinuous weak solutions.

The definition below includes the celebrated Barenblatt solution, which for $p>2$ is given as

$$
\mathcal{B}_{p}(x, t)= \begin{cases}t^{-\frac{n}{\lambda}}\left\{C-\frac{p-2}{2} \lambda^{\frac{1}{1-p}}\left(\frac{|x|}{t^{1 / \lambda}}\right)^{\frac{p}{p-1}}\right\}_{+}^{\frac{p-1}{p-2}}, & t>0 \\ 0, & t \leq 0\end{cases}
$$

when $(x, t) \neq(0,0)$. Here $\lambda=n(p-2)+p$, and $C$ is a positive constant. With the definition $\mathcal{B}_{p}(0,0)=0$, the Barenblatt solution is $p$-superparabolic in the whole $\mathbb{R}^{n} \times \mathbb{R}$, although

$$
\int_{-1}^{1} \int_{|x|<1}\left|\nabla \mathcal{B}_{p}(x, t)\right|^{p} d x d t=\infty .
$$

Definition 4.2. A function $u: \mathcal{O} \rightarrow \mathbb{R} \cup\{\infty\}$ is p-superparabolic if

(i) $u$ is lower semicontinuous,

(ii') $u$ is finite in a dense subset of $\mathcal{O}$, and

(iii) $u$ satisfies the comparison principle on each box $Q_{t_{1}, t_{2}}$ with closure in $\mathcal{O}$ : if $h \in C\left(\bar{Q}_{t_{1}, t_{2}}\right)$ is p-parabolic in $Q_{t_{1}, t_{2}}$ such that $h \leq u$ on the parabolic boundary of $Q_{t_{1}, t_{2}}$, then $h \leq u$ in $Q_{t_{1}, t_{2}}$.

We refer to $[\mathrm{KL}]$ for a detailed discussion on the properties of the $p$-superparabolic functions.

Let us next turn to the definition of viscosity solutions of (4.1). Due to the presence of the time derivative $u_{t}$ in the equation, we cannot exclude test-functions with vanishing spatial gradient $\nabla \varphi(x, t)$ at the point of touching like we did with the $p$-harmonic equation. As in the elliptic case, the equation is singular only in the range $1<p<2$, but we have chosen again not to distinguish between the two cases.

Definition 4.3. A function $u: \mathcal{O} \rightarrow \mathbb{R} \cup\{\infty\}$ is a parabolic viscosity $p$-supersolution if

(i) $u$ is lower semicontinuous,

(ii') $u$ is finite in a dense subset of $\mathcal{O}$, and

(iv) whenever $\left(x_{0}, t_{0}\right) \in \mathcal{O}$ and $\varphi \in C^{2}(\mathcal{O})$ are such that $u\left(x_{0}, t_{0}\right)=\varphi\left(x_{0}, t_{0}\right)$, $u(x, t)>\varphi(x, t)$ for $(x, t) \in \mathcal{O} \cap\left\{t<t_{0}\right\}$, and $\nabla \varphi(x, t) \neq 0$ if $x \neq x_{0}$, we have

$$
\limsup _{\substack{(x, t) \rightarrow\left(x_{0}, t_{0}\right) \\ t<t_{0}, x \neq x_{0}}}\left(\varphi_{t}(x, t)-\Delta_{p} \varphi(x, t)\right) \geq 0 .
$$

The concepts of parabolic viscosity p-subsolution and parabolic viscosity p-solution are defined analogously. Notice that the parabolic viscosity $p$-solutions are continuous by definition. As in the elliptic case, the precaution about $\nabla \varphi \neq 0$ can be ignored for $p \geq 2$.

What is to happen in the future will have no influence on the present time. This phenomenon, typical of parabolic equations, was taken into account in the definition 
above: the test-function is forced to be under the function $u$ only up to the time $t_{0}$ of testing. This yields the same concept as a definition with no emphasis on the special role of the time variable; cf. [Ju].

THEOREM 4.4. Let $1<p<\infty$. In a given domain, the $p$-superparabolic functions and the parabolic viscosity $p$-supersolutions are the same.

Corollary 4.5. Let $1<p<\infty$. A continuous function is a parabolic viscosity p-solution if and only if it is p-parabolic.

The proof of Theorem 4.4 is virtually the same as its elliptic counterpart. To show that a $p$-superparabolic function is a parabolic viscosity $p$-supersolution, one needs to consider space-time boxes instead of balls and use the comparison principle for $p$-superparabolic and $p$-subparabolic functions; see [KL]. For the converse, the comparison principle for viscosity solutions is needed (Theorem 4.10 below).

Lemma 4.6. Every p-superparabolic function $u$ is a parabolic viscosity p-supersolution.

Proof. We argue by contradiction and assume that there exist $\varphi \in C^{2}(\mathcal{O})$ and $r>0$ such that $u(0,0)=\varphi(0,0), u(x, t)>\varphi(x, t)$ for all $(x, t) \in \mathcal{O} \cap\{t<0\}$, $\nabla \varphi(x, t) \neq 0$ when $x \neq 0$, and

$$
\varphi_{t}(x, t)-\Delta_{p} \varphi(x, t)<0
$$

whenever $(x, t) \in Q_{r} \cup\{x \neq 0\}$, where $Q_{r} \equiv B_{r}(0) \times(-r, 0)$. Then for every nonnegative $\phi \in C_{0}^{\infty}\left(Q_{r}\right)$, we obtain using (4.3) and Gauss's theorem as in the proof of Lemma 3.4 that

$$
\begin{aligned}
& -\iint_{Q_{r}}|\nabla \varphi|^{p-2}\langle\nabla \varphi, \nabla \phi\rangle d x d t \\
& =\lim _{\rho \rightarrow 0}\left[\iint_{Q_{r} \backslash\{|x| \leq \rho\}} \phi\left(\Delta_{p} \varphi\right) d x d t-\iint_{Q_{r} \backslash\{|x| \leq \rho\}} \operatorname{div}\left(\phi|\nabla \varphi|^{p-2} \nabla \varphi\right) d x d t\right] \\
& \geq \lim _{\rho \rightarrow 0}\left[\iint_{Q_{r} \backslash\{|x| \leq \rho\}} \phi \varphi_{t} d x d t+\int_{-r}^{0} \oint_{|x|=\rho} \phi|\nabla \varphi|^{p-2}\left\langle\nabla \varphi, \frac{x}{\rho}\right\rangle d S d t\right] \\
& =-\iint_{Q_{r}} \phi_{t} \varphi d x d t .
\end{aligned}
$$

This implies that $\varphi$ is $p$-subparabolic in $Q_{r}$; see [KL]. To conclude, we proceed as in the elliptic case, and apply the comparison principle for $p$-superparabolic and $p$ subparabolic functions from [KL] to the functions $u$ and $\varphi+m$, where

$$
m=\inf _{\partial_{p a r} Q_{r}}(u-\varphi)>0 .
$$

This gives the desired contradiction.

Due to the fact that the time derivative $u_{t}$ appears as a linear term in (4.1), Theorem 4.7 below is easier to prove than the elliptic comparison principle, Theorem 2.7. In fact, the nonsingular case $p \geq 2$ follows from a very general result in [C]. Since the basic idea of the proof is quite similar to the one of the elliptic case, we will be somewhat sketchy.

TheOREM 4.7. Let $\Omega_{T}=\Omega \times(0, T)$, where $\Omega \subset \mathbb{R}^{n}$ is a bounded domain, and assume that $u$ is a parabolic viscosity p-subsolution and $v$ is a parabolic viscosity p-supersolution in $\Omega_{T}$. If $u \leq v$ on the parabolic boundary of $\Omega_{T}$, then $u \leq v$ in $\Omega_{T}$. 
Proof. For simplicity, we assume that $u$ is bounded from above and $v$ is bounded from below in $\bar{\Omega} \times[0, T]$. Since the proof is by contradiction, we assume that

$$
\sup _{\Omega_{T}}(u-v)>\sup _{\partial_{\text {par }} \Omega_{T}}(u-v),
$$

where $\partial_{\text {par }} \Omega_{T}$ denotes the parabolic boundary of $\Omega_{T}$. By using the standard trick of replacing $v$ by $v(x, t)+\frac{\varepsilon}{T-t}$ for small $\varepsilon>0$, we may assume that $v$ is a strict supersolution of (4.1) and $v(x, t) \rightarrow \infty$ as $t \rightarrow T$.

Let $\left(x_{j}, y_{j}, t_{j}, s_{j}\right)$ be a maximum point of

$$
w_{j}(x, y, t, s)=u(x, t)-v(y, s)-\Psi_{j}(x, y, t, s)
$$

relative to $\bar{\Omega} \times \bar{\Omega} \times[0, T]$. Here

$$
\Psi_{j}(x, y, t, s)=\frac{j}{q}|x-y|^{q}+\frac{j}{2}(t-s)^{2}, \quad q>\max \left\{\frac{p}{p-1}, 2\right\} .
$$

By (4.4) and Proposition 3.7 in [CIL], we have that $\left(x_{j}, y_{j}, t_{j}, s_{j}\right) \in \Omega \times \Omega \times(0, T) \times$ $(0, T)$ for $j$ large enough. We distinguish between two cases.

Case 1. $x_{j}=y_{j}$.

By the choice of the point $\left(x_{j}, y_{j}, t_{j}, s_{j}\right)$ we have

$$
v(y, s) \geq-\Psi_{j}\left(x_{j}, y, t_{j}, s\right)+\Psi_{j}\left(x_{j}, y_{j}, t_{j}, s_{j}\right)+v\left(y_{j}, s_{j}\right)
$$

for all $(y, s) \in \Omega \times[0, T]$; that is,

$$
\phi(y, s) \equiv-\Psi_{j}\left(x_{j}, y, t_{j}, s\right)+\Psi_{j}\left(x_{j}, y_{j}, t_{j}, s_{j}\right)+v\left(y_{j}, s_{j}\right)-\frac{1}{q+1}\left|y-y_{j}\right|^{q+1}
$$

is touching $v$ from below at $\left(y_{j}, s_{j}\right)$. Since $v$ is a strict supersolution of (4.1) and $x_{j}=y_{j}$, we obtain after straightforward computations

$$
0<\frac{\varepsilon}{\left(T-s_{j}\right)^{2}} \leq \limsup _{\substack{(y, s) \rightarrow\left(y_{j}, s_{j}\right) \\ s<s_{j}, y \neq y_{j}}}\left(\phi_{s}(y, s)-\Delta_{p} \phi(y, s)\right)=j\left(t_{j}-s_{j}\right) .
$$

Similarly, we see that

$$
\theta(x, t) \equiv \Psi_{j}\left(x, y_{j}, t, s_{j}\right)-\Psi_{j}\left(x_{j}, y_{j}, t_{j}, s_{j}\right)+u\left(x_{j}, t_{j}\right)+\frac{1}{q+1}\left|x-x_{j}\right|^{q+1}
$$

is a good test-function for $u$ at the point $\left(x_{j}, t_{j}\right)$, and hence

$$
0 \geq \liminf _{\substack{(x, t) \rightarrow\left(x_{j}, t_{j}\right) \\ t<t_{j}, x \neq x_{j}}}\left(\theta_{t}(x, t)-\Delta_{p} \theta(x, t)\right)=j\left(t_{j}-s_{j}\right)
$$

Subtracting (4.6) from (4.5) gives

$$
0<\frac{\varepsilon}{\left(T-s_{j}\right)^{2}} \leq j\left(t_{j}-s_{j}\right)-j\left(t_{j}-s_{j}\right)=0
$$

which is a contradiction. 
Case 2. $x_{j} \neq y_{j}$.

As in the elliptic case, we may use the definition with jets. Using the maximum principle for semicontinuous functions and Lemma 3.5 from [OS], we infer that there exist $X_{j}, Y_{j} \in S_{n}$ such that

$$
\begin{aligned}
\left(D_{t} \Psi_{j}, D_{x} \Psi_{j}, X_{j}\right) & \in \overline{\mathcal{P}}^{2,+} u\left(x_{j}, t_{j}\right), \\
\left(-D_{s} \Psi_{j},-D_{y} \Psi_{j}, Y_{j}\right) & \in \overline{\mathcal{P}}^{2,-} v\left(y_{j}, s_{j}\right),
\end{aligned}
$$

and

$$
X_{j} \leq Y_{j} .
$$

Here all the derivatives of $\Psi_{j}$ are evaluated at the point $\left(x_{j}, y_{j}, t_{j}, s_{j}\right)$. For the definition and properties of the parabolic jets $\mathcal{P}^{2,+} u$ and $\mathcal{P}^{2,-} v$ and their closures, we refer the reader to $[\mathrm{C}]$ and $[\mathrm{CIL}]$.

Let us now finish the proof. Since $u$ is a subsolution and $v$ is a strict supersolution, we obtain

$$
0<\frac{\varepsilon}{\left(T-s_{j}\right)^{2}} \leq-D_{s} \Psi_{j}+F\left(-D_{y} \Psi_{j}, Y_{j}\right)-D_{t} \Psi_{j}-F\left(D_{x} \Psi_{j}, X_{j}\right) \leq 0,
$$

which is a contradiction. Here the last inequality follows from (4.7) after we notice that

$$
\begin{aligned}
D_{t} \Psi_{j}(x, y, t, s) & =-D_{s} \Psi_{j}(x, y, t, s), \\
D_{x} \Psi_{j}(x, y, t, s) & =-D_{y} \Psi_{j}(x, y, t, s),
\end{aligned}
$$

by the choice of $\Psi_{j}$. This shows that (4.4) cannot hold, and we are done.

Remark 4.8. In [CGG], Chen, Giga, and Goto obtained a general comparison theorem for mean curvature flow-type equations. Those equations are singular at the points where the spatial gradient vanishes, but the nature of the singularity is different from that of the $p$-parabolic equation. Roughly speaking, the mean curvature flow equation has a bounded discontinuity at the points of singularity, whereas the $p$ parabolic equation behaves like $O\left(|\nabla u|^{p-2}\right)$ near those points.

We finish the paper with a brief discussion on another possible definition for viscosity solutions of (4.1) in the singular case $1<p<2$. This approach is due to Ishii and Souganidis [IS], and in connection with the $p$-parabolic equation it has been used by Ohnuma and Sato; cf. [OS].

Let us introduce some notation. We set

$$
\begin{gathered}
\mathcal{F}=\left\{f \in C^{2}([0, \infty)) \quad f(0)=f^{\prime}(0)=f^{\prime \prime}(0)=0, f^{\prime \prime}(r)>0 \text { for all } r>0\right. \text {, and } \\
\left.\lim _{x \rightarrow 0_{+}}\left(-\Delta_{p} f(|x|)\right)=0\right\}
\end{gathered}
$$

and

$$
\Sigma=\left\{\sigma \in C^{1}(\mathbb{R}) \sigma \text { is even, } \sigma(0)=\sigma^{\prime}(0)=0 \text {, and } \sigma(r)>0 \text { for all } r \neq 0\right\} .
$$

Definition 4.9. A function $\varphi \in C^{2}(\mathcal{O})$ is admissible if for any $(\hat{x}, \hat{t}) \in \mathcal{O}$ with $\nabla \varphi(\hat{x}, \hat{t})=0$ there are $\delta>0, f \in \mathcal{F}$, and $\sigma \in \Sigma$ such that

$$
\left|\varphi(x, t)-\varphi(\hat{x}, \hat{t})-\varphi_{t}(\hat{x}, \hat{t})(t-\hat{t})\right| \leq f(|x-\hat{x}|)+\sigma(t-\hat{t})
$$

for all $(x, t) \in B_{\delta}(\hat{x}) \times(\hat{t}-\delta, \hat{t}+\delta)$. 
Notice that if $\nabla \varphi \neq 0$, then $\varphi$ is automatically admissible. The idea of introducing the admissible class is, roughly speaking, to have a good a priori control on the behavior of a test-function at the singular points. For lack of a better terminology, we call the solutions defined using the class of admissible test-functions relaxed viscosity solutions.

Definition 4.10. A function $u: \mathcal{O} \rightarrow \mathbb{R} \cup\{\infty\}$ is a relaxed viscosity p-supersolution if

(i) $u$ is lower semicontinuous,

(ii') $u$ is finite in a dense subset of $\mathcal{O}$, and

(v) for all admissible $\varphi \in C^{2}(\mathcal{O})$ and all local minimum points $(x, t)$ of $u-\varphi$ in

$\mathcal{O}$

$$
\begin{cases}\varphi_{t}(x, t)-\Delta_{p} \varphi(x, t) \geq 0 & \text { if } \nabla \varphi(x, t) \neq 0 \\ \varphi_{t}(x, t) \geq 0 & \text { if } \nabla \varphi(x, t)=0\end{cases}
$$

We have taken the liberty to modify the definition given in [IS], [OS] in order to make a comparison with Definition 4.3 easier. In particular, the original definition of Ishii and Souganidis was formulated without the semicontinuity assumption (i), and thus it does not imply continuity for the solutions.

LEMMA 4.11. Every parabolic viscosity p-supersolution is a relaxed viscosity $p$ supersolution.

Proof. We argue by contradiction and assume that there exist an admissible testfunction $\varphi \in C^{2}(\mathcal{O})$ and $\left(x_{0}, t_{0}\right) \in \mathcal{O}$ such that $u-\varphi$ has a local minimum at $\left(x_{0}, t_{0}\right)$, $\nabla \varphi\left(x_{0}, t_{0}\right)=0$, and

$$
\varphi_{t}\left(x_{0}, t_{0}\right)<0
$$

Let $f \in \mathcal{F}, \sigma \in \Sigma$, and $\delta>0$ be such that

$$
\left|\varphi(x, t)-\varphi\left(x_{0}, t_{0}\right)-\varphi_{t}\left(x_{0}, t_{0}\right)\left(t-t_{0}\right)\right| \leq f\left(\left|x-x_{0}\right|\right)+\sigma\left(t-t_{0}\right)
$$

for all $(x, t) \in B_{\delta}\left(x_{0}\right) \times\left(t_{0}-\delta, t_{0}+\delta\right)$. Following the ideas in [IS], we approximate $\sigma$ by a sequence $\sigma_{k} \in C^{2}(\mathbb{R})$ satisfying

$$
\left\{\begin{array}{l}
\sigma_{k}(0)=\sigma_{k}^{\prime}(0)=0 \text { for each } k=1,2, \ldots \\
\sigma_{k}(r) \rightarrow \sigma(r), \sigma_{k}^{\prime}(r) \rightarrow \sigma^{\prime}(r) \quad \text { locally uniformly }
\end{array}\right.
$$

and we denote

$$
\begin{aligned}
\phi(x, t) & =u\left(x_{0}, t_{0}\right)+\varphi_{t}\left(x_{0}, t_{0}\right)\left(t-t_{0}\right)-2 f\left(\left|x-x_{0}\right|\right)-2 \sigma\left(t-t_{0}\right), \\
\phi_{k}(x, t) & =u\left(x_{0}, t_{0}\right)+\varphi_{t}\left(x_{0}, t_{0}\right)\left(t-t_{0}\right)-2 f\left(\left|x-x_{0}\right|\right)-2 \sigma_{k}\left(t-t_{0}\right) .
\end{aligned}
$$

Observe that (4.9) implies that $u-\phi$ has a strict local minimum at $\left(x_{0}, t_{0}\right)$. Since $\sigma_{k} \rightarrow \sigma$ locally uniformly, we can find a sequence $\left(x_{k}, t_{k}\right) \rightarrow\left(x_{0}, t_{0}\right)$ such that $u-\phi_{k}$ has a local minimum at $\left(x_{k}, t_{k}\right)$. Moreover, by modifying $\phi_{k}$ if necessary, we may assume that this local minimum is, in fact, strict. Hence $\phi_{k}$ can be used as a testfunction in Definition 4.3, and we obtain

$$
\limsup _{\substack{(x, t) \rightarrow\left(x_{k}, t_{k}\right) \\ t<t_{k}, x \neq x_{0}}}\left(\left(\phi_{k}\right)_{t}(x, t)-\Delta_{p} \phi_{k}(x, t)\right) \geq 0
$$


for each $k \in \mathbb{N}$. However, a direct computation yields

$$
\left(\phi_{k}\right)_{t}(x, t)-\Delta_{p} \phi_{k}(x, t)=\varphi_{t}\left(x_{0}, t_{0}\right)-2 \sigma_{k}^{\prime}\left(t-t_{0}\right)-2^{p-1} \Delta_{p} f\left(\left|x-x_{0}\right|\right)<0
$$

if $(x, t)$ is sufficiently close to $\left(x_{0}, t_{0}\right)$ and $x \neq x_{0}$. Here we used (4.8), the definition of $\mathcal{F}$, and the fact that $\sigma_{k}^{\prime}(0)=0$. This contradiction shows that the antithesis was wrong, and the lemma is now proved.

In [OS], Ohnuma and Sato proved a comparison principle for the relaxed viscosity $p$-supersolutions and subsolutions. In the light of Lemmas 4.6 and 4.11, this means that relaxed viscosity $p$-supersolutions satisfy (p-iii) in Definition 4.2 , and hence they are precisely the parabolic viscosity $p$-supersolutions.

Acknowledgments. We are grateful to Eero Saksman for pointing out an error in an earlier version of the manuscript. We thank the anonymous referee for the careful reading of the manuscript and useful suggestions and comments.

\section{REFERENCES}

[B] G. I. Barenblatt, On selfsimilar motions of compressible fluids in a porous medium, Prikl. Mat. Mekh., 16 (1952), pp. 679-698 (in Russian).

[CGG] Y. Chen, Y. GigA, AND S. Goto, Uniqueness and existence of viscosity solutions of generalized mean curvature flow equations, J. Differential Geom., 33 (1991), pp. 749-786.

[C] M. G. CRAndall, Viscosity solutions: A primer, in Viscosity Solutions and Applications (Montecatini Terme, 1995), Lecture Notes in Math. 1660, Springer-Verlag, Berlin, 1997, pp. 1-43.

[CIL] M. G. Crandall, H. Ishit, and P-.L. Lions, User's guide to viscosity solutions of second order partial differential equations, Bull. Amer. Math. Soc. (N.S.), 27 (1992), pp. 1-67.

[CKSS] M. Crandall, M. Kocan, P. Soravia, And A. Swiech, On the equivalence of various weak notions of solutions of elliptic PDEs with measurable ingredients, in Progress in Elliptic and Parabolic Partial Differential Equations, Pitman Res. Notes Math. Ser. 350, A. Alvino and P. Buoncore, eds., Longman, Harlow, UK, 1996, pp. 136-162.

[DB] E. DiBenedetto, Degenerate Parabolic Equations, Springer-Verlag, New York, 1993.

[DB2] E. DiBenedetto, $C^{1+\alpha}$-local regularity of weak solutions of degenerate elliptic equations, Nonlinear Anal., 7 (1983), pp. 827-850.

[FIT] I. Fukuda, H. Ishit, And M. Tsutsumi, Uniqueness of solutions to the Cauchy problem for $u_{t}+u \Delta u+\gamma|\nabla u|^{2}=0$, Differential Integral Equations, 6 (1993), pp. 1231-1252.

[HKM] J. Heinonen, T. Kilpeläinen, And O. Martio, Nonlinear Potential Theory of Degenerate Elliptic Equations, Oxford University Press, Oxford, UK, 1993.

[IS] H. IshiI AND P. E. Souganidis, Generalized motion of noncompact hypersurfaces with velocity having arbitrary growth on the curvature tensor, Tohoku Math. J. (2), 47 (1995), pp. 227-250.

[Je] R. JENSEN, Uniqueness of Lipschitz extensions: Minimizing the sup norm of the gradient, Arch. Ration. Mech. Anal., 123 (1993), pp. 51-74.

[Je2] R. JENSEn, Uniformly elliptic PDEs with bounded, measurable coefficients, J. Fourier Anal. Appl., 2 (1996), pp. 237-259.

[Ju] P. JuUtinen, On the definition of viscosity solutions for parabolic equations, Proc. Amer. Math. Soc., 129 (2001), pp. 2907-2911.

[JLM] P. Juntinen, P. Lindqvist, And J. Manfredi, The $\infty$-eigenvalue problem, Arch. Ration. Mech. Anal., 148 (1999), pp. 89-105.

[KL] T. KILPELÄINEn AND P. LindqVist, On the Dirichlet boundary value problem for a degenerate parabolic equation, SIAM J. Math. Anal., 27 (1996), pp. 661-683.

[Le] J. LEWIS, Regularity of the derivatives of solutions to certain elliptic equations, Indiana Univ. Math. J., 32 (1983), pp. 849-858.

[Li] P. Lindqvist, On the definition and properties of p-superharmonic functions, J. Reine Angew. Math., 365 (1986), pp. 67-79.

[Lie] G. M. Lieberman, Boundary regularity for solutions of degenerate elliptic equations, Nonlinear Anal., 12 (1988), pp. 1202-1219. 
[LMS] P. Lindqvist, J. Manfredi, And E. Saksman, Superharmonicity of nonlinear ground states, Rev. Mat. Iberoamericana, 16 (2000), pp. 17-28.

[OS] S. Ohnuma And K. SATo, Singular degenerate parabolic equations with applications to the p-Laplace diffusion equation, Comm. Partial Differential Equations, 22 (1997), pp. 381-411.

[Ur] N. Ural'tseva, Degenerate quasilinear elliptic systems, Zap. Nauchn. Sem. Leningrad. Otdel. Mat. Inst. Steklov. POMI, 7 (1968), pp. 184-222 (in Russian). 